\title{
Hyphenated Techniques, an Important Tool for Force Degradation Study
}

\section{Rushikesh Shirgaonkar, Aniket Lomate and Sachin A Pishawikar*}

Department of Pharmaceutical Quality Assurance, Bharati Vidyapeeth College of

Pharmacy, Navi Mumbai, Maharashtra, India

*Corresponding Author: Sachin A Pishawikar, Department of

Pharmaceutical Quality Assurance, Bharati Vidyapeeth College of Pharmacy,

Navi Mumbai, Maharashtra, India.
Received: July 22, 2020

Published: August 28, 2020

(C) All rights are reserved by Sachin A

Pishawikar., et al.

\begin{abstract}
The pharma market is getting flooded with many new drug formulations as well as fixed dose combination formulations in which different API are there. Each of this is going to contain different types of impurities as well as they are bound to undergo process of degradation by different mechanisms leading to formation of degradation products. All of these are going to be present as impurities. Though the impurities will be within limit in accordance to regulatory authorities, still in case of disease like diabetes and hyper tension the patient is going to be on medicine for rest of his life hence bound to get over exposure to these. Some of which may cause adverse effects like cytotoxic and genotoxic. Hence indeed there is need for identification. In previous era it was ok with regulatory authorities if components in formulation were $99 \%$ pure. But know they are insisting on identification of that remaining $1 \%$.

Though there is challenge in front of analytical chemists, use of sophisticated instruments for analysis of impurities has increased and has made task little easier. Scientist are working towards discovering different types of methods whereby accurate results can be obtained. Hyphenated techniques have received tremendous attention as it is been seen as the principal means to solve complex analytical problems. The combined power of separation techniques like chromatography in combination with spectroscopic techniques has been predominantly used over the years for both quantitative and qualitative analysis of unknown compounds. The hyphenated techniques have provided analytical chemist a tool to come over the challenge associated with impurity profiling and determination of safety and efficacy of API. Among all hyphenated techniques, the most exploited techniques for impurity profiling of drugs are Liquid Chromatography (LC)-Mass Spectroscopy (MS), LC-NMR, LC-NMR-MS, GC-MS and LC-MS. This reveals the need and scope of impurity profiling of drugs in pharmaceutical research.
\end{abstract}

Keywords: Liquid Chromatography (LC); Mass Spectroscopy (MS); Hyphenated Techniques; Force Degradation

\section{Introduction}

Hyphenated techniques is combination of two techniques in which one is used to carry out isolation or separation of component preferably chromatographic technique and other is used for identification of separated component using techniques like mass, NMR or IR spectroscopy. Chromatographic procedure plays important role in separation of components in complex mixture in pure chemical form while spectroscopic procedure give selective information for identification by carrying comparative study with standards or library spectra.

Few decked back scientist Hirschfeld has introduced the term "hyphenation" with refer to the on-line combination of a separation technique and one or more spectroscopic detection techniques. 
In recent years, hyphenated techniques have received tremendous attention as it is been seen as the principal means to solve complex analytical problems. The combined power of separation technologies with spectroscopic techniques has been predominantly used over the years for both quantitative and qualitative analysis of unknown compounds from complex natural product extracts or fractions as well as the use has started taking place for carrying out impurity profiling as well as qualitative and quantitative analysis of impurity and allied products [1-7].

To obtain structural information leading to the identification of the compounds present in a crude sample, liquid chromatography (LC), a high-performance liquid chromatography (HPLC), gas chromatography (GC), or capillary electrophoresis (CE) are attached with spectroscopic detection techniques like Fourier-transform infrared (FTIR), photodiode array (PDA), UV-VISIBLE absorbance or fluorescence emission, mass spectroscopy (MS) and nuclear magnetic resonance spectroscopy (NMR). It has resulted in the various new modern hyphenated techniques like CE-MS, GC-MS, LC-MS and LC-NMR. The hyphenation does not always have to be between two techniques; the coupling of separation and detection techniques can involve more than one separation or detection techniques like LC-PDA-MS, LC-MS-MS, LC-NMR-MS, LCPDA-NMR-MS.

The development of various hyphenated techniques has provided the natural product researchers with extremely powerful new tools that provide excellent effective separation as well as acquisition of on-line complementary spectroscopic data on an LC or GC peak of interest within a complex mixture [8-14].

The market of medicine is getting flooded with many formulations in which combination of different API is there. Each of this can undergo process of degradation and lead to formation of cytotoxic, genotoxic and other types of impurities. The hyphenated techniques has provided analytical chemist a tool to come over the challenge associated with impurity profiling and determination of safety and efficacy of API.

The various sources through which impurity can get incorporated in pharmaceutical products is, use of specific reactants and reagents in reactions, heavy metals, ligands, catalysts, other materials like filter aids, charcoal, used during manufacturing and degraded end products generated during storage of manufactured bulk drugs. The generation of degradant may occur due to pro- cesses like hydrolysis, photolytic cleavage, oxidative degradation, decarboxylation, enantiomeric impurity, and so on due to exposure to various atmospheric conditions. The various pharmacopoeias like British Pharmacopoeia, United State Pharmacopoeia and Indian Pharmacopoeia have slowly started incorporating limits of allowable levels of impurities present in APIs or formulations.

Hence it has become need of the hour to use various hyphenated techniques in identification and quantitation of impurities. This reveals the need and scope of impurity profiling of drugs in pharmaceutical research.

\section{Systematic approach for impurity profiling}

Impurity profiling is systematic approach for identifying, elucidation of structure and qualitative and quantitative determination of impurities and degradation products in bulk drug materials as well as pharmaceutical formulations.

For any API achieving $100 \%$ purity is note possible. Initially if there was a mention that the compound is $99 \%$ pure it was enough. But nowadays various regulatory authorities have started insisting on detection of composition of remaining $1 \%$. This is due to few facts like in treatment of diseases like if a person is diagnosed with hypertension, disease like diabetes, for rest of his life he or she has to be put on medicine. The drugs or formulations prescribed to him or her have to be used for very long time. Though the presence of impurities is going to be limited still prolong exposure to even small quantity may lead to features like genotoxicity and cytotoxicity. Hence it is need of the hour that proper impurity profiling for all formulations has to be done.

Discovery of various hyphenated techniques has turned out to be blessing for analytical chemist as in fixed dose combination type of formulation qualitative analysis with impurity profiling has become easier.

Why there is need for identification and quantitation of impurity?

- Impurities may lead to causing incompatibility with other substances.

- Impurities can lead to lowering of the shelf life of the substances.

- Impurities may cause difficulties during formulations and use of the substances. 
- Sometimes Impurities may change the physical and chemical properties of the substances.

- Therapeutic effect can be reduced.

- May lead to showing toxic effect after a certain period.

- Are going to be injurious when present above certain limits.

- It may cause changes in odor, color, taste of the substance.

Hence identification, quantification and control of impurities in API and the drug product are critical in drug development.

\section{Approach used in impurity profiling}

During or after manufacturing of bulk drugs various types of impurities might be generated due to hydrolysis, photolytic cleavage, oxidative degradation, decarboxylation, thermal, enantiomeric impurity, and so on due to exposure to various atmospheric conditions. Hence under systematic approach impurity profiling is done by carrying out force degradation study.

Basics for carrying out force degradation and reasoning related to use of specific degradation condition

For degradation study following

A systematic approach now has been developed for stress testing of pharmaceutical products which can generate the degradation in most efficient way. Many different types of stress conditions are employed for the degradation study. These includes acid hydrolysis by using $\mathrm{HCl}$ having concentration range between 0.01 to $1 \mathrm{~N}$ and alkali hydrolysis using $\mathrm{NaOH}$ in the range of 0.01 to $1 \mathrm{~N}$, while for oxidative degradation use of $3 \% \mathrm{H}_{2} \mathrm{O}_{2}$ and photolytic degradation use UV light having specific flux as mentioned in ICH Q1B is done. For acid, alkali hydrolysis, and oxidation, the study period is $1 \mathrm{~h}$ whereas for light study period is $24 \mathrm{~h}$. All stress conditions employed for forced degradation studies are initially carried out at room temperature $\left(25^{\circ} \mathrm{C}\right)$. It should include the effect of temperatures in $10^{\circ} \mathrm{C}$ increments (e.g. $50^{\circ} \mathrm{C}, 60^{\circ} \mathrm{C}$ etc.) above that of accelerated testing, humidity (e.g. $75 \% \mathrm{RH}$ or greater), acid-base hydrolysis, oxidation, and photolysis. Stress testing should induce not more than 5 - 15\% degradation of the main compound. Stress conditions employed for forced degradation study as per ICHQ1A (R2).

\section{Reason for hydrolytic degradation study using $\mathrm{HCl}$ and $\mathrm{NaOH}$}

Once the bulk manufacturing of API has been done, it might not be used immediately for formulation development. During its storage as well as manufacturing and storage of various types of formulations API is not going to get exposed to acid or base. Still for degradation study by:

On what basis hydrolytic study using acid and base can be justified?

During storage pure API as well as its formulation is going to get exposed to various atmospheric conditions. Sometime due to absorption of moisture certain structural features of API would undergo process of breaking down and may lead to formation of ionic components. Due to this acidic or basic properties may get imparted, which may cause further degradation. Hence hydrolysis is one of the common chemical reactions used in force degradation study over a wide range of $\mathrm{pH}$. Hydrolysis is a chemical process that includes decomposition of a chemical compound by reaction with water. Hydrolytic study under acidic and basic condition involves catalysis of ionisable functional groups present in the molecule. Acid or base stress testing involves forced degradation of a drug substance by exposure to acidic or basic conditions which generates primary degradant in desirable range. The type and concentrations of acid or base used in study depends on the stability of the drug substance. Hydrochloric acid with concentration range between $0.01-1 \mathrm{M}$ is used for acid hydrolysis while sodium hydroxide or potassium hydroxide with concentration range $0.01-1 \mathrm{M}$ is used for base hydrolysis are considered as suitable reagents for hydrolysis. Along with this effect of temperature is also taken it to consideration.

Stress testing trial normally starts with use of room temperature and if there is no degradation, elevated temperature of 50 $70^{\circ} \mathrm{C}$ is applied. Stress testing should not exceed more than 7 days. The degraded sample is then neutralized using suitable acid, base or buffer, to avoid further decomposition [15-20].

\section{Oxidation conditions}

Hydrogen peroxide is widely used for oxidative stress degradation study of drug substance as most suitable agent, but other oxidizing agents such as metal ions, oxygen, and radical initiators like, azobisisobutyronitrile, AIBN can also be used. Selection of an oxidizing agent, its concentration, and conditions to be applied depends on the stability of drug substance. Generally, the solution of drug is subjected to hydrogen peroxide solution having concentration range between $0.1-3 \%$. The study is done at neutral $\mathrm{pH}$ and room temperature for seven days or till up to a maximum $20 \%$ 
degradation could potentially generate [22]. The oxidative degradation of drug substance involves an electron transfer mechanism to form reactive anions and cations. Amines, sulfides and phenolic structural features are susceptible to electron transfer oxidation to give $\mathrm{N}$-oxides, hydroxylamine, sulfones and sulfoxide [23]. The functional group with labile hydrogen like benzylic carbon, allylic carbon, and tertiary carbon or $\alpha$-positions with respect to hetro atom are susceptible to oxidation to form hydro peroxides, hydroxide or ketone.

\section{Photolytic conditions}

The photo stability testing of drug substances must be carried out to find out whether exposure to a light result in unacceptable change in the form of formation of photolytic impurities or not. Photo stability studies are performed to generate primary degradants of drug substance by exposure to UV or fluorescent conditions. Some of the recommended conditions for photostability testing are described in ICH guidelines. Samples of drug substance and solid/liquid drug product has to be exposed to a minimum of 1.2 million $\mathrm{lx} h$ and $200 \mathrm{~W} \mathrm{~h} / \mathrm{m}^{2}$ light. The most commonly accepted wavelength of light is in the range of $300-800 \mathrm{~nm}$. The maximum illumination recommended is 6 million $\mathrm{lx}$ h. Light stress conditions may induce photo oxidation by free radical mechanism. Presence of structural features in the form of functional groups like carbonyls, nitro aromatic, $\mathrm{N}$-oxide, alkenes, aryl chlorides, weak $\mathrm{C}-\mathrm{H}$ and $\mathrm{O}-\mathrm{H}$ bonds, sulfides and polyenes in the drug are likely to introduce photosensitivity degradation.

\section{Thermal conditions}

Thermal degradation (e.g. dry heat and wet heat) should be carried out at more strenuous conditions than recommended ICH Q1 Accelerated testing conditions. Samples of solid-state drug substances and drug products should be exposed to dry and wet heat, while liquid drug products should be exposed to dry heat. Studies may be conducted at higher temperatures for a shorter period [22]. Effect of temperature on thermal degradation of a substance is studied using Arrhenius equation:

$\mathrm{k}^{1} / 4 \mathrm{AeEa}=\mathrm{RT}$

Where, $\mathrm{k}$ represents specific reaction rate, A represents frequency factor, Ea represents energy of activation, $\mathrm{R}$ is gas constant (1.987 cal/deg mole) and $\mathrm{T}$ is absolute temperature. Use of tem- perature range between $40-80^{\circ} \mathrm{C}$ is done. The heating is done in chamber or use of calibrated thermostatic dry heat oven is done.

\section{Regulatory guidelines}

Before planning for force degradation study it has become essential to study recommended guidelines. Most important are the guidelines provided by ICH. Different categories of the guidelines given by ICH for forced degradation studies are as follows:

- ICH Q1A: Stability testing of new drug substances and products,

- ICH Q1B: Photo stability testing of new drug substances and products,

- ICH Q2B: Validation of analytical procedures: Methodology.

ICH Q1A (Stress testing): Recommended conditions for performing forced degradation studies on drug substances and drug products. The recommendations are to inspect the results of temperature (above that for accelerated testing, i.e. $>50^{\circ} \mathrm{C}$ ), humidity (75\% relative humidity), oxidation and photolysis. Wide $\mathrm{pH}$ range should be considered in the testing of solution or suspension. Ultimately the stability-indicating method developed by these samples [15-20].

\section{ICH Q1B}

These are recommended for assessing the photo stability of drug substances as well as drug products. Solid or in solution/suspension, photo stability testing can be performed. The samples are further used in development of a stability indicating method.

\section{ICH Q2B}

These guidelines give information about how to carry out validation of developed analytical method.

\section{ICH Q3A (R2)}

Both with respect chemistry and safety the identification of each and every impurity is required. In relation to chemistry, classification and identification of impurities, how by force degradation can lead to their formation and if possible developing analytical method is discussed. report creation, catalogue of impurities in the specification and a concise discussion of analytical methods. For safety measures whether the impurities are within limit or not has to be checked. 
Main objective of forced degradation study is:

- Developing and studying degradation pathways of drug substances and drug products.

- Study the chemical properties associated with the drug molecules.

- To carry out structural elucidation of degradation products.

- To find out and try to resolve stability-related problems

- To establish the intrinsic stability of a drug substance in the formulation.

- To reveal the degradation mechanisms of the drug substance and drug product.

- To distinguish degradation products that is related to drug products from those that are generated from non-drug product in a formulation.

To generate stability indicating nature of a developed method.

1. To produce more stable formulations. It also helps in determining the expiry date of a particular formulation.

2. To generate a degradation profile similar to that of what would be observed in a formal stability study under ICH conditions.

Use of hyphenated analytical techniques for impurity profiling

Due to application of various degradation conditions generation of degradation products with different physical and chemical properties is going to take place. Hence each one of the product has to be first separated and then identified. The most common hyphenated analytical technique used for monitoring forced degradation study is HPLC with UV and/or MS spectroscopy. Now a days ultra-performance liquid chromatography (UPLC), UPLC-specific photodiode array (PDA) and quadruple time-of-flight mass spectrometry (QTOF-MS/MS) like techniques are being introduced for combining the chromatographic speed, resolution, and sensitivity of detection for peak purity, mass balance and identification of degradation products. This has lead to faster detection and identification of degradation products within short period of time and less time is required to develop stability-indicating methods [24-30]. As generation and detection of degradation products using suitable analytical technique has become faster it has provided analytical chemist a tool to establish degradation pathway deter- mination of shelf lives of drug products. It has plaid significant role in formulation development process and the testing can be applied different stages of pharmaceutical development is given in table 1.

\begin{tabular}{|l|c|}
\hline Development Stage & $\begin{array}{c}\text { Purpose of carrying out stress } \\
\text { testing }\end{array}$ \\
\hline Pre-formulation & $\begin{array}{c}\text { Useful in selection of compounds } \\
\text { and } \\
\text { Compatible excipients, Formulation } \\
\text { Optimization, making selection of } \\
\text { proper packaging }\end{array}$ \\
\hline $\begin{array}{l}\text { Formulation } \\
\text { registration batches } \\
\text { and manufacturing }\end{array}$ & $\begin{array}{c}\text { To carry out stability study, } \\
\text { impurity profiling, finding } \\
\text { degradation pathway and structural } \\
\text { elucidation of degraded products, } \\
\text { determination of shelf life, selection } \\
\text { of packing material and instructing } \\
\text { storage condition. }\end{array}$ \\
\hline
\end{tabular}

Table 1: Information provided by stress testing at various stages.

\section{Conclusion}

Force degradation and impurity profiling study has become mandatory due to directives of regulatory authorities. Carrying out force degradation study has become challenge of analytical chemist. In force degradation, along with original compound various degradation products are going to be formed. Separation and identification of each of the component is must. Here development of number of hyphenated technique like GC-MS, LC-MS, LC-FTIR, LC-NMR etc have made it easy task.

\section{Bibliography}

1. Yoshioka S and Stella VJ. "Stability of drugs and dosage forms". Kluwer Academic Publisher: New York (2002).

2. Carstensen JT and Rhodes CT. "Drug Stability Principles and Practices". Marcel Dekker: New York (2000).

3. International Conference on Harmonization, ICH Q1A (R2), Stability Testing of New Drug Substances and Products, Geneva (2003).

4. International Conference on Harmonization, ICH Q1B, Photo stability testing of new drug substances and products, Geneva (1996). 
5. FDA, Guidance for Industry: Stability Testing of Drug Substances and Drug Products. Rockville, MD (1998).

6. WHO Guidelines for stability testing of Active Pharmaceutical Ingredients and finished pharmaceutical products (2009).

7. Branch SK. "Guidelines from the International Conference on Harmonization". Journal of Pharmaceutical and Biomedical Analysis 38.5 (2005): 798-805.

8. Singh S and Bakshi M. "Development of stability-indicating assay methods". Journal of Pharmaceutical and Biomedical Analysis 28.6 (2002): 1011-1040.

9. Singh S and Bakshi M. "Guidance on conduct of stress tests to determine inherent stability of drugs". Pharmaceutical Technology 24 (2000): 1-14.

10. Rourick RA., et al. "Predictive strategy for the rapid structure elucidation of drug degradants". Journal of Pharmaceutical and Biomedical Analysis 14.12 (1996): 1743-1752.

11. Reynolds DW. "Available guidance and best practices for conducting forced degradation studies". Pharmaceutical Technology 26.2 (2002): 48-56.

12. Alsante KM., et al. "A stress testing benchmarking study". Pharmaceutical Technology 27.2 (2003): 60-72.

13. Khan H., et al. "Stability testing of pharmaceutical products: Comparison of stability testing guidelines". Current Pharmaceutical Analysis 6.2 (2010): 140-148.

14. Khan H., et al. "Validated UPLC/Q-TOF-MS method for simultaneous determination of aceclofenac, paracetamol, and their degradation products in tablets". Journal of Liquid Chromatography and Related Technologies 35.1 (2012): 109-128.

15. International Conference on Harmonization, ICH Q3A (R2), Impurities in New Drug Substances, Geneva (2003).

16. International Conference on Harmonization, ICH Q3B (R2), Impurities in New Drug Products, Geneva, (2003).

17. Ahuja S. "Impurities evaluation of pharmaceuticals". Marcel Dekker, New York (2006).
18. Qiua F and Norwood DL. "Identification of pharmaceutical impurities". Journal of Liquid Chromatography and Related Technologies 30.5 (2007): 877-935.

19. Roy J. "Pharmaceutical impurities- A review". AAPS PharmSciTech 3.2 (2002): 1-8.

20. Rahman N., et al. "Importance of impurity analysis in pharmaceutical products". Accreditation and Quality Assurance 11.2 (2006): 69-74.

21. Dong MW., et al. "Liquid chromatographic considerations for high sensitivity impurity and stability testing of pharmaceuticals". Journal of Liquid Chromatography and Related Technologies 13.11 (1990): 2135-2160.

22. Wong AW and Datla A. In Hand Book of Pharmaceutical Analysis by HPLC, Ahuja S, Dong MW, Edition. Elsevier Academic Press: San Diego (2005): 335-358.

23. Ahuja S and Scypinski S. "Hand Book of Modern Pharmaceutical Analysis". Harcourt Science and Technology: San Diego (2001).

24. Lee H. "Pharmaceutical applications of liquid chromatography coupled with mass spectrometry (LC/MS)". Journal of Liquid Chromatography and Related Technologies 28.7 (2005): 11611202.

25. Lee H., et al. "Identification and control of impurities for drug substance development using LC/MS and GC/MS". Journal of Liquid Chromatography and Related Technologies 31.15 (2008): 2235-2252.

26. Vyas VK., et al. "Recent advances in characterization of impurities-Use of hyphenated LC-MS technique". Current Pharmaceutical Analysis 6.4 (2010): 299-306.

27. Ravi Sankar S. Text Book of Pharmaceutical Analysis, Rx Publications: India (2001).

28. Swartz ME. "UPLC: An Introduction and Review". Journal of Liquid Chromatography and Related Technologies 28.1 (2005): 1253-1263.

29. Novakova L., et al. "Advantages of application of UPLC in pharmaceutical analysis". Talanta 68.3 (2006): 908-918. 
30. Plumb R., et al. "Ultra performance liquid chromatography coupled to quadrupole-orthogonal time-of-flight mass spectrometry". Rapid Communications in Mass Spectrometry 18.19

(2004): 2331-2337.

\section{Assets from publication with us}

- Prompt Acknowledgement after receiving the article

- Thorough Double blinded peer review

- Rapid Publication

- Issue of Publication Certificate

- High visibility of your Published work

Website: www.actascientific.com/

Submit Article: www.actascientific.com/submission.php

Email us: editor@actascientific.com

Contact us: +919182824667 